\title{
Conocimiento docente sobre evaluación de los aprendizajes y su aplicación en la práctica de evaluación en los centros escolares públicos de Educación Básica en la Zona Oriental
}

\author{
Isabel Hernández de Guevara \\ Máster en Métodos y Técnicas de Investigación Social \\ Investigadora \\ Universidad de Oriente \\ Email: iherandez@univo.edu.sv
}

Recepción: 11-11-2014 / Aceptación: 11-01-2015

\section{Resumen}

El estudio realizado por la Universidad de Oriente, a través del convenio con el MINED (Ministerio de Educación) ${ }^{1}$, tuvo como objetivo determinar si el conocimiento que tiene el docente sobre evaluación de los aprendizajes, influye en las prácticas de esta naturaleza que él realiza. La metodología utilizada para recabar la información fue de tipo cuantitativa, descriptiva y correlacional.

Los resultados confirmaron que la funcionalidad y los agentes de la evaluación; así como la evaluación por competencias, influyen y promueven la aplicación de esta en los centros escolares públicos. Por ello, el proceso pedagógico no se puede concebir sin la evaluación del aprendizaje. Si se imparte un contenido, la forma más efectiva de saber cómo ha llegado el conocimiento a los estudiantes es a través de la evaluación, en cualquiera de sus formas posibles, (exámenes tradicionales, pruebas objetivas u otros).

Palabras claves: Conocimiento, evaluación de los aprendizajes, aplicación, competencias, funcionalidad, agentes de evaluación.

\begin{abstract}
The study carried out by the Universidad de Oriente through the covenant with MINED (Ministry of Education $)^{1}$ had as objective to determine if the knowledge that the teacher has about learning evaluations influences in the practices that they carry out. The methodology used to dig in the information was qualitative, descriptive, and correlational.

The results confirmed that the functionality and the agents of the evaluation, as well as the evaluation for competencies influence and foster the application of it in the public schools. For it, the pedagogical process cannot be conceived without the learning evaluation. If a content is given, the more effective way to know how the knowledge has come to the students is through the evaluation in any of its possible forms (traditional tests, objective tests and others).
\end{abstract}

Key words: knowledge, learning evaluation, applications, competencies, functionality, agents of evaluation

1. De aquí en adelante, la autora también se referirá a esta entidad mediante sus siglas.

1. From here on, the author will refer to this entity by its acronym 


\section{Introducción}

La evaluación de los aprendizajes constituye un importante campo de investigación en permanente desarrollo, así como un conjunto de prácticas que tienen lugar en diferentes ámbitos del sistema educativo. A ella se le debe otorgar una atención particular, debido a que se le considera un procedimiento esencial para el mejoramiento de la calidad educativa.

Durante largo tiempo se ha constatado que, la evaluación de estudiantes y/o del aprendizaje se ha limitado esencialmente al proceso cognitivo. La evaluación al servicio del aprendizaje busca que se evalúe el desarrollo de competencias en los educandos; a fin de orientar a los docentes hacia una valoración, orientación y fortalecimiento del aprendizaje de los alumnos, por medio de la evaluación por competencias. Esta última debe entenderse como el conjunto de habilidades, conocimientos, medios y actitudes integradas que permite dar respuesta a nuevos problemas, dentro de las distintas situaciones de todos los ámbitos de la vida personal, social y profesional; permitiendo a los alumnos actuar de forma activa.

En la actualidad, los programas de estudio están diseñados para evaluar por competencias, debido a que explican los dominios conceptuales, procedimentales y actitudinales; sin olvidar que la evaluación de los aprendizajes de los estudiantes no es simplemente una ac- tividad técnica. Esta constituye un elemento clave en la calidad del aprendizaje. Sin embargo, algunos docentes continúan evaluando tradicionalmente. Por su parte, la evaluación sumativa está centrada en los objetivos del dominio conceptual (cognitivos), relegando a planos secundarios los otros dominios.

\section{Evaluación de los aprendizajes}

La evaluación de los aprendizajes es una de las tareas de mayor complejidad que realizan los docentes, tanto por el proceso que implica como por las consecuencias que tiene emitir juicios sobre los logros de aprendizaje de los alumnos.

Acercarse y profundizar en la evaluación de los aprendizajes solo es posible si se concientizan las emociones que ello involucra: la forma en que se enseña y en la que aprenden los alumnos; los valores implicados, las consecuencias que pueden existir al respecto de la inclusión y la exclusión y; sobre todo, responder honestamente si se confía en la capacidad de aprender de todos y cada uno de los estudiantes.

En la actualidad, se insiste en la importancia de que el propósito de la evaluación en el aula sea mejorar el aprendizaje y desempeño de los alumnos, mediante la creación constante de mejores oportunidades para aprender. Todo esto a partir de los resultados que ellos obtienen en cada evaluación presentada durante el ciclo escolar. 
En resumen, significa dejar atrás el papel sancionador y el carácter exclusivamente conclusivo o sumativo de la evaluación de aprendizajes, por uno más interesado en conocer por qué los alumnos se equivocan o tienen fallas, con la finalidad de identificar las causas para ayudarlos a superarlas. En eso consiste lo que se ha denominado enfoque formativo de la evaluación de los aprendizajes.

Con la frase "evaluar para aprender", no solo se hace referencia a los alumnos y sus aprendizajes, sino también se remite a la posibilidad de que todos los que participan en el proceso de evaluación aprendan de sus resultados. Es decir, también se dirige a los educadores y los maestros, quienes mediante los procesos de evaluación tienen la oportunidad de mejorar la enseñanza; adecuándola a las necesidades de aprendizaje de sus alumnos. De esta forma, la evaluación también es una herramienta para mejorar la práctica docente.

\section{La evaluación: caracterización general}

Al hacer un recorrido no exhaustivo a lo largo de la historia de la Evaluación, se observa que los chinos (hace más de 4000 años), ya practicaban pruebas a los ciudadanos que pretendían alcanzar la función pública. Posteriormente, Sócrates y diferentes filósofos griegos (siglo V a. C.), empleaban cuestionarios evaluativos en sus prácticas.

Pero es hasta el siglo XIX en que se fundan las bases para el establecimiento de un modelo de evaluación para la escuela tradicional.
Man, en Estados Unidos hacia 1845, administró una evaluación por medio de test para estudiar el rendimiento de las escuelas de Boston y el tipo de educación de los estudiantes. El test estuvo centrado en los resultados de los alumnos, y no en los programas, ni en los métodos educativos. Por su parte, Rice (1898) realizó un estudio sobre Ortografía a 33000 alumnos (durante los años 1887 a 1898); llegando a la conclusión de que no se habían producido adelantos en la enseñanza. Ese trabajo consistió en la primera evaluación de un programa educativo en Norteamérica. Al mismo tiempo de la exigencia evaluativa surgió el proceso de industrialización, el cual mostró nuevos requerimientos en el área de la educación. Con las ideas de Fayol, referentes al papel administrativo, publicadas en su Libro "Administración general e industria" (1906), surgen los entendidos en diferentes áreas como son el control, la organización y la planificación educativa.

El sentido moderno sobre la evaluación se basa en el florecimiento del positivismo, el empirismo, y los métodos estadísticos utilizados en el estudio de la diversidad humana. A la pedagogía también contribuyen el surgimiento, la divulgación y el empleo de los test psicológicos.

El rasgo "g" sobre la inteligencia general fue descubierto en 1904, por el psicólogo inglés Spearman. La primera prueba de inteligencia fundamentada en patrones, analogías y habi- 
lidades razonadas fue establecida por el francés Binet en 1905. El concepto de coeficiente intelectual, entendido como la correspondencia entre la edad mental y la edad cronológica, fue determinado por el alemán Stern hacia 1912. Todos esos test prestan a los docentes una herramienta de cuantificación de las capacidades y del aprendizaje de los discentes en el sistema educativo, por medio de la estadística descriptiva y su expansión a otros ámbitos educativos. Es así como aparece en la educación, de la mano de Tyler, la evaluación científica, bajo un modelo cuantitativo y de mentalidad tecnocrática. Sus contribuciones aún se reflejan en la actualidad, a pesar de que se basan en fundamentos psicopedagógicos ya superados.

En 1950, Tyler señala que con el fin de medir los aprendizajes de los alumnos es determinar con anterioridad los objetivos de forma cuantificable, para comparar los objetivos propuestos, los resultados obtenidos; y conocer su grado de consecución. De esta forma se establece la programación basada en objetivos, la cual emplea por primera vez el término evaluación, entendida como "el proceso que permite determinar en qué grado han sido alcanzados los objetivos educativos propuestos".

En la década de los años 60 aumenta el interés hacia esta disciplina, surgiendo la educación permanente y global, así como el interés por el fracaso escolar. Ello lleva a prestar atención hacia la evaluación tanto educativa como empresarial, con el aparecimiento de instrumentos de evaluación que fueran fiables y útiles. Para Cronbach (1963), la evaluación se ha de entender como una fase de "recogida y uso de información para tomar decisiones sobre un programa educativo". Así la evaluación deja atrás el hecho de ser entendida como un instrumento de control y medida, con la intención de llevar a cabo una valoración al final de un proceso, pasando a ser vista como un medio que posibilita la retro alimentación del proceso educativo.

Scriven (1967) señala que se tiene que situar el grado de valor y mérito del objeto evaluado. Según este autor, previo al establecimiento del grado alcanzado por los objetivos, hay que verificar la adecuación de los mismos al fin perseguido. Con ello la evaluación es entendida como un proceso de "enjuiciamiento sistemático del valor o mérito de un objeto". Se establecen las nociones de programas entendidos como evaluación formativa y evaluación sumativa, y se toman a los objetos como evaluación intrínseca y extrínseca.

En la década de los años 70 se establece un cambio bajo las ideas de Piaget y la concepción constructivista del aprendizaje. Surgen las opciones cualitativas que priorizan el proceso y el uso de procedimientos antropológicos. Se establecen las ideas de evaluación iluminativa, democrática, etnográfica, entre otras. Cesa la exclusividad de la aplicación 
de la evaluación a los alumnos y se desarrolla - entre otros elementos - en los centros, en los profesores y en los programas educativos (Morales Artero, 2001).

El concepto de evaluación formativa se amplía en 1980, gracias a Cronbach, en función de la idea de que, aunque se pretenda medir los resultados, la evaluación siempre tendría que seguir esta idea. Para Blanco (1996: 42), "La evaluación es el enjuiciamiento comparativo, corrector y continuo del progreso del alumno, a partir de unos datos recogidos”. Así es como entiende este autor la evaluación en el marco de la reforma de la LOGSE. De este modo, se establece un recorrido por las diferentes definiciones de evaluación las cuales se pueden agrupar en cuatro grandes categorías en la línea de Tejada (1997):

Definiciones que ponen la atención en el logro de objetivos alcanzados por los alumnos, en su evaluación y en el interés por los resultados.

1. Tyler (1950: 69): "Proceso que determina hasta qué punto se han conseguido los objetivos educativos."

2. Lafourcade (1972: 21): "Etapa del proceso educacional que tiene por fin controlar de modo sistemático en qué medida se han conseguido los objetivos que se hubieran especificado con antelación.”
3. Gronlund (1973: 2): "Proceso sistemático para determinar hasta qué punto alcanzan los alumnos los objetivos de la educación.”

4. Mager (1975: 20): "Acto de comparar una medida con un estándar y emitir un juicio basado en la comparación."

Estas definiciones se ajustan únicamente, en la evaluación de los alumnos, a un nivel explícito o implícito, resaltándose la inquietud por el alcance de los objetivos como ente básico de la evaluación al final del proceso formativo; es decir, centrado en la evaluación sumativa.

\section{Metodología}

La investigación fue de carácter descriptivo porque se estableció la relación directa entre la variable independiente y dependiente, puesto que lo que se pretendía investigar era el grado de conocimiento que tiene el docente de la evaluación de los aprendizajes y su aplicación en la práctica de evaluación, en el aula en los centros escolares públicos de Educación Básica de la Zona Oriental.

Con una población total de 8836 alumnos, se tomó una muestra de 192 docentes; diseñándose un cuestionario como instrumento, tomando como base los indicadores de cada una de las variables en estudio. Este se conformó de un total de 25 ítems, dejando la posibilidad de respuesta abierta en algunas de ellas, la cual se tomó en cuenta dentro de la formulación de conclusiones y sus respectivas recomendaciones. 


\section{Resultados}

Al consultar si durante su formación como docente, ¿considera que tuvo una adecuada formación en el tema de evaluación de los aprendizajes?

\section{Opinión sobre la evaluación de los aprendizajes}

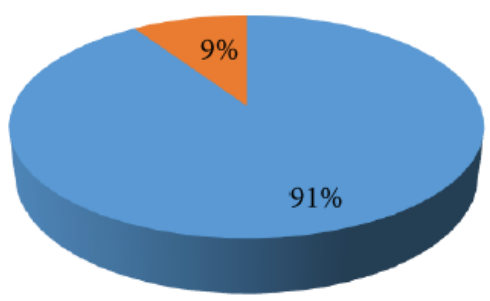

Figura 1. Gráfico sobre evaluación de los aprendizajes.

El 76.6\% de ellos contestó que sí, argumentando que ahí fue donde aprendieron a aplicar los diferentes tipos de evaluación; mientras que otros respondieron que sí se les explicó cómo hacerlo, pero lo olvidaron y no lo ponen en práctica. Por su parte, el 22.9\% contestó negativamente, aduciendo que para algunos docentes la teoría es una cosa y la práctica otra, concluyendo que necesitan un refuerzo. Finalmente, el 0.5\% no respondió.

Para la pregunta: ¿Ha recibido capacitaciones de parte del MINED u otra institución sobre evaluación de los aprendizajes?

\section{Opinión sobre capacitaciones del MINED}

Docentes

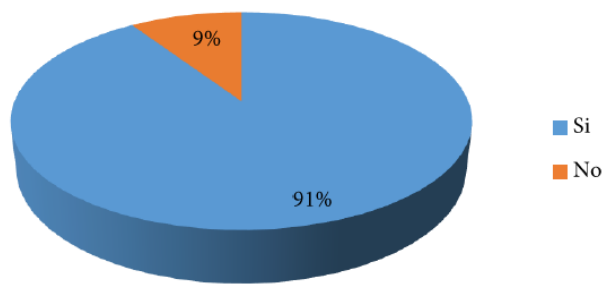

Figura 2. Gráfico respecto a capacitación del MINED.

El $85.4 \%$ de los encuestados declararon que toman capacitaciones en esta entidad y en la Escuela Superior de Maestros (ESMA). Ellos manifestaron que antes las recibían al inicio o fin de año, de acuerdo a los lineamientos; pero que hoy en día casi no reciben formación. El 14.1\% que contestó que no, declaró que sería bueno que las capacitaciones se enfocaran hacia los nuevos docentes; mientras que a los que ya están formados como tales, deberían recibir capacitación de parte de un docente capaz en la materia. Un porcentaje del 0.5\% no contestó. Si bien el porcentaje mayor afirma haber recibido capacitación sobre evaluación de los aprendizajes, es importante prestar atención a los comentarios vertidos por algunos docentes.

En otra interrogante en donde se les cuestionó sobre si han recibido capacitación sobre evaluación por competencias, los resultados fueron muy reveladores. El 76.6\% declaró que sí, señalando que recibieron este tipo de capacitación cuando se realizaron los cambios de programas de estudio. Aseguraron que la for- 
mación no fue muy profunda, debido a que lo único que se realizó fue compartir contenidos que ya se dominaban; o comentar otros aspectos, pero a nivel educativo del estudiante con dominios por cada asignatura. Mientras tanto, el $22.9 \%$ reveló que no han recibido ningún tipo de formación sobre la temática, y que lo han aprendido es porque preguntan a docentes de otras instituciones; además de que cuando los convocan a alguna capacitación, la explicación que dan al respecto es poca. Nuevamente, el 0.5\% no contestó.

\section{Opinión sobre evaluación por competencias}

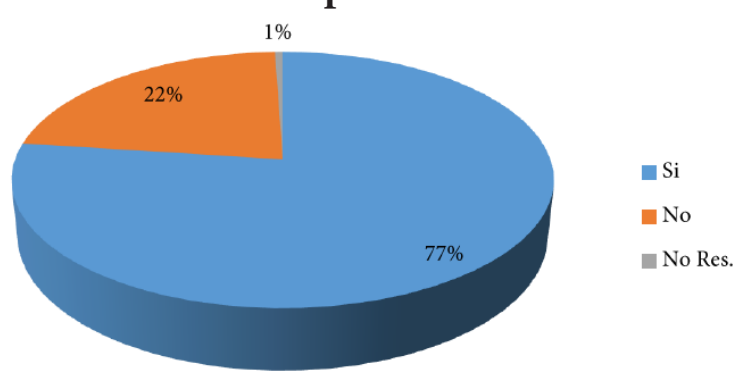

Figura 3. Gráfico sobre evaluación por competencias.

Para la pregunta ¿Considera que la evaluación diagnóstica refleja las fortalezas y debilidades de alumnos/as?, las respuestas de los encuestados se reflejan en la siguiente figura.

\section{Opinión sobre evaluación diagnóstica}

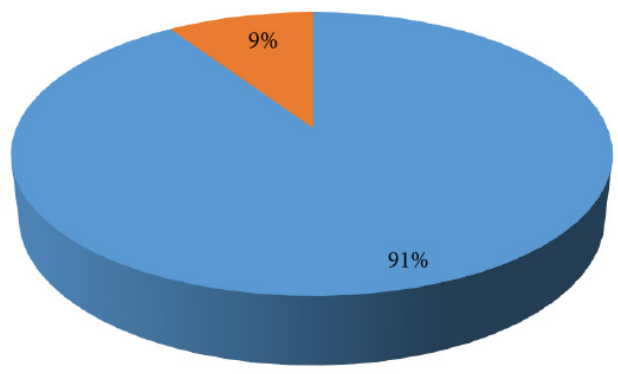

Figura 4. Gráfico sobre evaluación diagnóstica.
Al preguntar si la evaluación diagnóstica refleja las fortalezas y debilidades de los alumnos, el $90.6 \%$ contesto que sí, comentando que esto les permite conocer el punto de partida para la acción, detectar debilidades en las diferentes materias; conocer los saberes previos de los estudiantes, hacer las adecuaciones correspondientes y planificar de acuerdo a las necesidades que estos presentan. Sin embargo, un 9.4\% manifestó lo contrario, ello debido a que hay estudiantes que son cambiantes; no son sinceros al contestar o no tienen claridad de su vivencia.

\section{Discusión}

En la investigación se hizo una revisión exhaustiva en el temario de la Asociación de Universidades Privadas de El Salvador (AUPRIDES), para verificar si existía algún estudio relacionado con la temática, encontrándose un estudio de la Universidad de Oriente (UNIVO), con el tema "Efectividad del proceso de evaluación de los aprendizajes para mejorar la calidad de enseñanza en la asignatura de estudios sociales y cívica en alumnos/ as de tercer ciclo de educación básica de los centros escolares del distrito 12-10 de la ciudad de San Miguel". Ese trabajo posee cierto grado de similitud con la actual investigación, debido a que uno de los problemas más comunes en el campo educativo del Nivel Básico, es la carencia de una metodología precisa para evaluar el proceso de formación de alumnos. Si bien es cierto que las evaluaciones se suelen 
realizar, estas adolecen de una planificación rigurosa que apunta a resultados.

Sin embargo, existe otra investigación realizada en la Universidad Pedagógica Nacional Francisco Morazán (UPNFM), de la república de Honduras, con el tema "La evaluación de los aprendizajes basada en competencias en la enseñanza universitaria”.

En ella se propone un sistema de evaluación de los aprendizajes que constituye un aporte de gran utilidad práctica, debido a que este es el proceso más vital de la formación profesional. Un sistema de evaluación de alta calidad permite orientar, de manera correcta, los di- ferentes aspectos de un curso; al mismo tiempo que brinda a los estudiantes una retroalimentación apropiada. Asimismo, cuando los procedimientos son correctos, los estudiantes pueden confiar en la calidad de su formación, y los empleadores pueden confiar en la capacidad de los estudiantes calificados.

Esta investigación es una primera aproximación a la percepción sobre la evaluación de los aprendizajes y su aplicación en la práctica de evaluación de los centros escolares públicos de Educación Básica en la Zona Oriental. Los resultados deben verse como un punto de partida para nuevas investigaciones que se pretendan hacer y no como algo concluido.

\section{Referencias}

Abigail, H., Celia, M. y Ana, F. (s.f.). Evaluación Continua con un Enfoque por Competencias: Mejorando las políticas y las Prácticas en El Salvador. Recuperado de: www.equip123.net/docs/ e2-EvaluaciónContinua.pdf

Lourdes, V. G. (2006). Evaluación del Aprendizaje para promover el desarrollo de competencia. Bilbao: Universidad de Deusto.

Ministerio de Educación. MINED. (1999). Lineamientos para la Evaluación de los Aprendizajes en Educación Parvularia, educación Básica y educación Media. San Salvador. El Salvador.

Ministerio de Educación. MINED. (2007). Evaluación al Servicio del Aprendizaje. San Salvador, El Salvador.

Ministerio de Educación. MINED. (2008). Evaluación al servicio del Aprendizaje. San Salvador. El Salvador.

Morales Artero, J. J. (2001). La Evaluación en el Área de Educación Visual y Plástica en la ESO. Cataluña, España.

Secretaría de Educación Pública. (2013). La evaluación durante el ciclo escolar. México. 